Check for updates

Cite this: Chem. Sci., 2017, 8, 8078

\title{
Bis(aminothiolato)nickel nanosheet as a redox switch for conductivity and an electrocatalyst for the hydrogen evolution reaction $\uparrow$
}

\author{
Xinsen Sun, ${ }^{a}$ Kuo-Hui Wu, ${ }^{a}$ Ryota Sakamoto, (D) ${ }^{\text {a }}$ Tetsuro Kusamoto, (D) ${ }^{a}$ \\ Hiroaki Maeda, ${ }^{a}$ Xiaojuan $\mathrm{Ni}^{\text {, }}$, Wei Jiang, ${ }^{\text {b }}$ Feng Liu, ${ }^{\text {b }}$ Sono Sasaki, ${ }^{c d}$ \\ Hiroyasu Masunaga ${ }^{e}$ and Hiroshi Nishihara (D) *a
}

A $\pi$-conjugated coordination nanosheet comprising bis(aminothiolato)nickel (NiAT) moieties was synthesized by the reaction of $\mathrm{Ni}(\mathrm{acac})_{2}$ with 1,3,5-triaminobenzene-2,4,6-trithiol at liquid-liquid and gas-liquid interfaces. The sheet thickness could be controlled down to a single layer $(0.6 \mathrm{~nm})$. Selected area electron diffraction and grazing incidence $X$-ray diffraction analyses indicated the formation of a flat crystalline sheet with a kagome lattice stacked in a staggered alignment. NiAT was reversibly interconverted to a bis(iminothiolato)nickel (NilT) nanosheet by the chemical $2 \mathrm{H}^{+}-2 \mathrm{e}^{-}$reaction, which was accompanied by a drastic change in electrical conductivity from $3 \times 10^{-6}$ to $1 \times 10^{-1} \mathrm{~S} \mathrm{~cm}^{-1}$. This change in conductivity was explained by the difference in band structures between NiAT and NiIT. NiAT acted as an efficient electrocatalyst for the hydrogen evolution reaction, showing strong acid durability and an onset overpotential of $-0.15 \mathrm{~V}$.

Received 16th June 2017

Accepted 2nd October 2017

DOI: $10.1039 / c 7 s c 02688 a$

rsc.li/chemical-science research. ${ }^{28-41}$ Recently, various functionalities of CONASHs have been demonstrated. ${ }^{\mathbf{4 2 - 4 7}}$

2D materials based on the bis(dithiolato)metal complex motif and its analogues comprise important series in CONASHs. Their square-planar geometry is ideal for constructing 2D frameworks. Our first report $\mathrm{t}^{48}$ has stimulated their variations, in combination with metal ions $\left(\mathrm{Ni}^{\mathrm{II}}, \mathrm{Pd}^{\mathrm{II}}\right.$, and $\left.\mathrm{Cu}^{\mathrm{I}}\right)$ with aromatic organic ligands (triphenylenehexaol, ${ }^{\mathbf{4 9}, 50}$ benzenehexathiol, ${ }^{48,51-53}$ triphenylenehexaamine,$^{50,54-57}$ triphenylenehexathiol, $^{58,59}$ and benzenehexaamine $\left.{ }^{60}\right)$. They exhibit high electrical conductivity due to strong charge delocalization in the plane, ${ }^{53-57,61}$ some of them been theoretically predicted to be $2 \mathrm{D}$ topological insulators. ${ }^{62}$ Moreover, these CONASHs can also act as electrocatalysts: for example, the hydrogen evolution reaction (HER) was catalysed by the bis(dithiolato)metal type of CONASHs. ${ }^{\mathbf{5 8 , 6 3 , 6 4}}$

Herein, we add a new aspect in the bis(dithiolato)metal type of CONASHs, by creating a crystalline $\pi$-conjugated CONASH comprising bis(aminothiolato)nickel (NiAT, Fig. 1) synthesized by the interfacial reaction of 1,3,5-triaminobenzene-2,4,6trithiol (L) in water and bis(2,4-pentanedionato)nickel(II) $\left(\mathrm{Ni}(\mathrm{acac})_{2}\right)$ in $\mathrm{CH}_{2} \mathrm{Cl}_{2}$. We recently reported the synthesis of a bis(iminothiolato)nickel (NiIT) nanosheet (Fig. 1), ${ }^{65}$ although the reaction conditions were different. NiAT has composition and structure similar to those of NiIT, but different coordination modes with each other. The similarity allows NiAT and NiIT to be interconvertible reversibly via the chemical $2 \mathrm{H}^{+}-2 \mathrm{e}^{-}$ reaction per nickel complex unit, while the difference induces a drastic change in electrical conductivity. This change is explained by the obvious difference in band structures between 


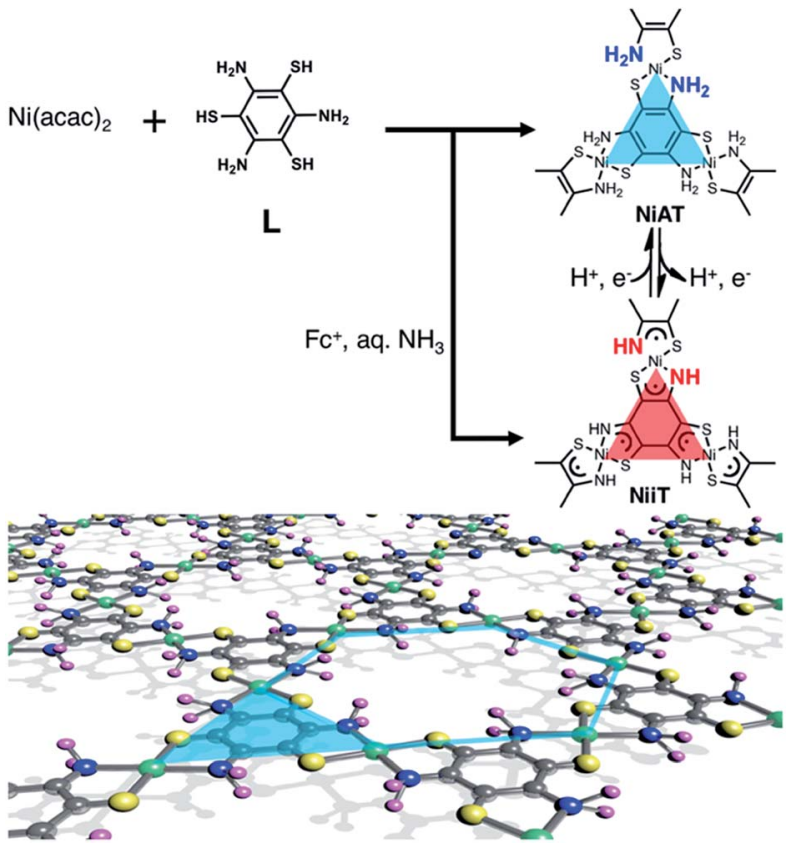

Fig. 1 Schematics and chemical structures of NiAT and NilT. Grey: C: yellow: S; blue: $\mathrm{N}$; purple: $\mathrm{H}$; green: $\mathrm{Ni}$.

NiAT and NiIT. We also find that NiAT exhibited high, durable catalytic activity for the HER.

\section{Results and discussion}

Typical fabrication processes of multi-layered and singlelayered NiAT via an interfacial reaction are shown in Fig. 2a and $b$, respectively. The multi-layered sheets were obtained at the liquid-liquid interface using an aqueous solution of $\mathbf{L}(1$

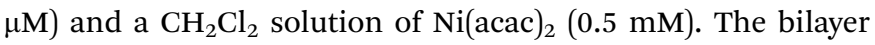
solution was stood at room temperature under argon for 8 days, and a light brown-coloured thin film formed at the liquid/liquid interface. The film was cleaned by repeatedly replacing each solution with pure solvent. The film was vertically transferred onto a flat silicon(111) crystal plate chemically modified with 1,1,1,3,3,3-hexamethyldisilazane [HMDS/Si(111)].

Optical microscopy revealed yellow colored film with lateral lengths of more than $50 \mu \mathrm{m}$ (Fig. 1c) and field-emission scanning electron microscopy (FE-SEM) of the film revealed flat and layered film morphologies (Fig. 1d). Atomic force microscopy (AFM) showed a flat, thin film façade with a thickness of $12 \mathrm{~nm}$ (Fig. 2e), which contained $c a$. 30 layers, based on the thickness of a single layer on [HMDS/Si(111)] of $0.6 \mathrm{~nm}$ and the interlayer distance of NiAT of $0.42 \mathrm{~nm}$. Wrinkles and folded sections were observed close to the edge of sheet, suggesting a uniform, pliable sheet material.

Single-layer NiAT was fabricated by a gas-liquid interfacial reaction (Fig. 2b). A tiny amount of $\mathrm{Ni}(\mathrm{acac})_{2}$ in $\mathrm{CH}_{2} \mathrm{Cl}_{2}$-EtOAc $(10: 1 \mathrm{v} / \mathrm{v})$ was spread onto an aqueous solution of $\mathbf{L}(35 \mu \mathrm{M})$ under argon. Composition of highly extensible ethyl acetate let the organic solution spread on water surface immediately, leading to rapid evaporation of solvent. After evaporation of the
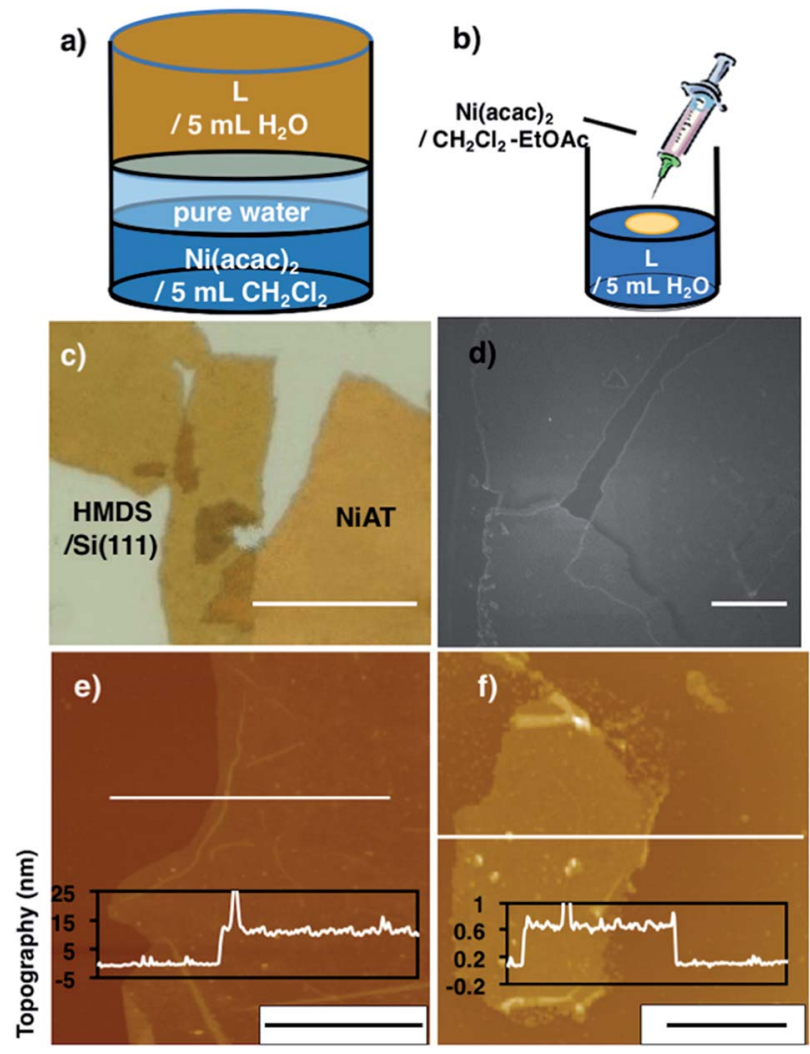

Fig. 2 (a) Schematics of the liquid-liquid interfacial synthesis of multilayer NiAT. (b) Schematics of the gas-liquid interfacial synthesis of single-layer NiAT. (c) Optical microscope images of multi-layer NiAT on HMDS/Si(111). Scale bars represent $50 \mu \mathrm{m}$. (d) FE-SEM images on HMDS/Si(111) of multi-layer NiAT. Scale bars represent $50 \mathrm{~nm}$. (e) AFM image on HMDS/Si(111) and its cross-section analysis along the white line of the multi-layer. Scale bars represent $10 \mu \mathrm{m}$. (f) AFM image on HMDS/Si(111) and its cross-section analysis along the magenta line along the white line of the single-layer. Scale bars represent $2 \mu \mathrm{m}$. AFM images of $(e)$ and $(f)$ including height distribution bar with roughness analysis along magenta lines (Fig. S1 $\uparrow$ ).

organic solvents, the coordination reaction proceeded at the gas-liquid interface to form an ultrathin film, which was transferred onto HMDS/Si(111). Fig. 2f shows that the film was $0.6 \mathrm{~nm}$ thick, corresponding to a single layer.

The chemical structure of the metal complex unit in the film was identified by attenuated total reflectance (ATR)-IR spectroscopy. The multi-layered film showed no S-H stretching vibration signal around $2500 \mathrm{~cm}^{-1}$, whereas this signal was observed for $\mathbf{L}$ (Fig. S2 $\dagger$ ), indicating that all the thiol groups of $\mathbf{L}$ participated in coordinating to the Ni centre. ${ }^{48}$ The amino N-H showed two bands ascribed to antisymmetric and symmetric stretching vibrations, whereas the imino $\mathrm{N}-\mathrm{H}$ exhibited only one $\mathrm{N}-\mathrm{H}$ stretching vibration band. The spectrum of the film contained two peaks at 3270 and $3380 \mathrm{~cm}^{-1}$, confirming the presence of the $-\mathrm{NH}_{2}-\mathrm{Ni}$ motif in NiAT. The previously reported NiIT film showed a single peak at $3270 \mathrm{~cm}^{-1}$ (Fig. 3a).

X-ray photoelectron spectroscopy (XPS) of NiAT detected N, $\mathrm{S}$, and $\mathrm{Ni}$ in a ratio of $1.98: 2.07: 1$ which was consistent with the ideal ratio of $2: 2: 1$ and indicated the quantitative formation of nickel complex units in the nanosheets (Fig. 3b-d). 

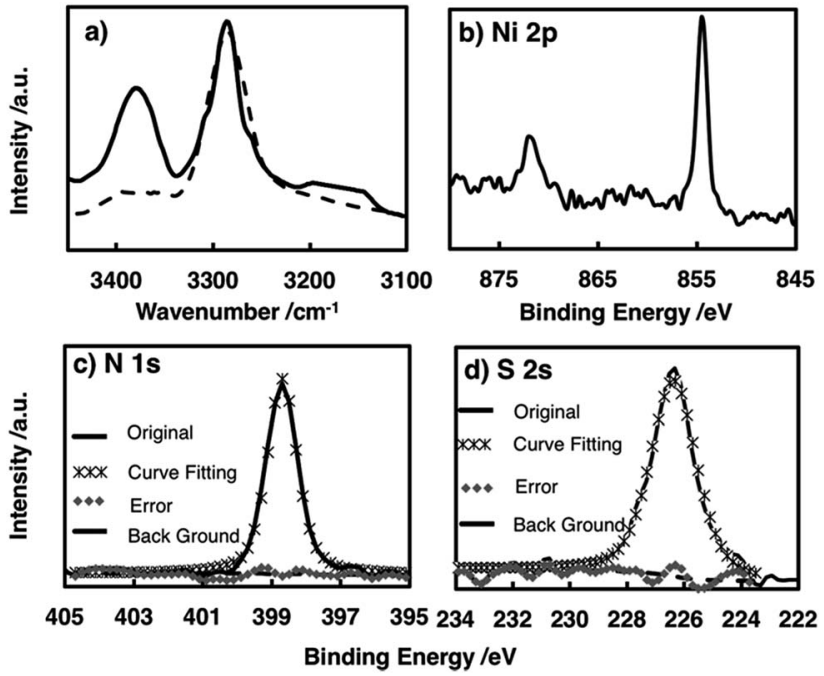

Fig. 3 (a) IR spectra in the $\mathrm{N}-\mathrm{H}$ stretching vibration region of NiAT (solid line) and NilT (dashed line). Narrow-scan XPS focusing on (b) the $\mathrm{Ni} 2 \mathrm{p}$ region, (c) the $\mathrm{N} 1 \mathrm{~s}$ region, and (d) the $\mathrm{S} 2 \mathrm{~s}$ region.

For reference, XPS spectra of $\mathbf{L}$, the NiIT nanosheet, and the neutral mononuclear complexes, bis(1-aminobenzene-2-thiolato)nickel(II) (NiAT-M) and bis(1-iminobenzene-2-thiolato) nickel(II) (NiIT-M), ${ }^{66}$ were also obtained (Fig. S3-S6 and Table $\mathrm{S} 1 \dagger)$. Two Ni 2p peaks at binding energies of 855 and $873 \mathrm{eV}$ for NiAT were assigned to $\mathrm{Ni} 2 \mathrm{p}_{3 / 2}$ and $\mathrm{Ni} 2 \mathrm{p}_{1 / 2}$, respectively (Fig. 3b). The N 1s peak for NiAT was observed at $398.7 \mathrm{eV}$ (Fig. 3c), slightly lower than that of NiIT at $399.9 \mathrm{eV}$ (Fig. S6 and Table S1 $\dagger$ ), which was similar to the comparison of NiAT-M and NiIT-M. Only one S 2s peak appeared at $226.5 \mathrm{eV}$ for NiAT (Fig. 3d), indicating that the nickel complex unit was in a neutral oxidation state. In conclusion, the NiAT film contained only one type of bis(aminothiolato)nickel moiety.

The crystal structure of multi-layered NiAT was analysed using diffraction techniques. High-resolution transmission electron microscopy (HR-TEM) showed its sheet-like morphology (Fig. 4a), and selected-area electron diffraction (SAED) found a hexagonal diffraction pattern (Fig. $4 \mathrm{~b}$ and $\mathrm{S} 6 \dagger$ ), implying a hexagonal lattice with a cell length of $1.41 \mathrm{~nm}$. Grazing incidence X-ray diffraction (GIXD) analysis using synchrotron radiation $(\lambda=1.00 \AA)$ found prominent peaks (Fig. 4d) for NiAT that matched the diffraction pattern of the staggered stacking structure $(P \overline{3}, a=b=1.405 \mathrm{~nm}$ and $c=$ $0.84 \mathrm{~nm}$ ) rather than that of the eclipsed stacking structure (absence of an in-plane intense peak at $2 \theta=13.7^{\circ}$ ) (Fig. $4 \mathrm{e}$ and $\mathrm{S} 7 \dagger$ ). Additionally, multiple interlayer distance patterns were subjected to both staggered and eclipsed stacking structures to identify that the interlayer distance of $0.42 \mathrm{~nm}$ of staggered stacking structure agrees with the experimental observation (Fig. S7a, $\dagger$ examples of simulation using different interlayer distance). Moreover, slipped-parallel (AB) orientation pattern simulations were performed by fixing an interlayer separation of $0.42 \mathrm{~nm}$ with different stacking angels, which again supported the staggered stacking (Fig. S7b, $\dagger$ examples of simulation using different stacking angels). By comparing the
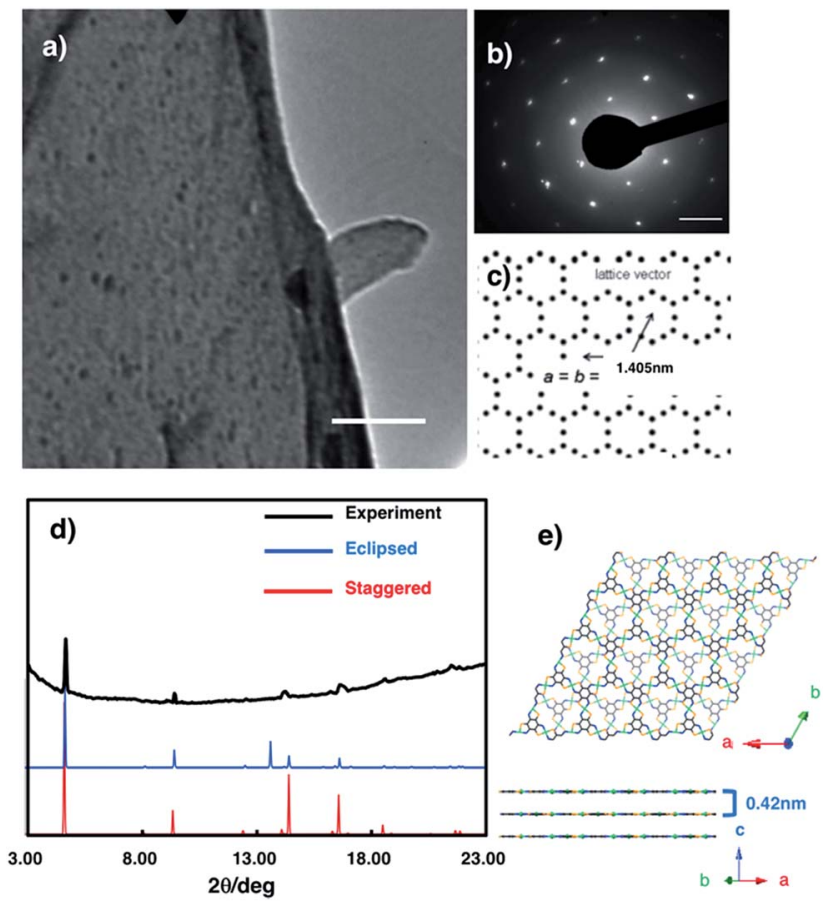

Fig. 4 (a) HR-TEM images of NiAT. Scale bars represent $500 \mathrm{~nm}$. (b) SAED pattern of NiAT. (c) Hexagonal two-dimensional lattice that gives the SAED pattern. Scale bar represents $2 \mathrm{~nm}^{-1}$. (d) Experimental and simulated GIXD patterns of NiAT. (e) Model structures of the staggered stacking structure.

observed GIXD profile with the simulated results, the sharp peaks at $2 \theta=4.7^{\circ}, 9.4^{\circ}, 12.5^{\circ}$, and $21.7^{\circ}$ were identified as the in-plane $100,200,310$, and 510 diffractions, respectively. GIXD indicated that the present nanosheet had a crystalline hexagonal lattice with an in-plane distance of $1.41 \mathrm{~nm}$ and interlayer distance of $0.42 \mathrm{~nm}$.

The NiAT-M and NiIT-M complexes are reversibly interconverted by a $2 \mathrm{H}^{+}-2 \mathrm{e}^{-}$reaction; ${ }^{66}$ therefore, interconversion of NiAT and NiIT was examined. The conversion of NiAT to NiIT was performed by treating NiAT suspended in water with a base $\left(\mathrm{NEt}_{3}\right)$ followed by adding ferrocenium tetrafluoroborate as an oxidizing agent. The conversion was reversed by the addition of acetic acid followed by the reducing agent, decamethylcobaltocene. This was confirmed directly by monitoring the characteristic $\mathrm{N}-\mathrm{H}$ stretching signals in the IR spectra (Fig. S8 $\dagger$ ). The double $\mathrm{N}-\mathrm{H}$ stretching peaks changed to a single peak upon the chemical oxidation of NiAT, indicating the formation of NiIT, and the double peaks reappeared upon the chemical reduction of NiIT, forming NiAT. Overall, these results demonstrate that NiAT and NiIT are chemically interconvertible without decomposition or a change in shape.

We previously found that pelletized NiIT showed a semiconducting nature with the activation energy of $41 \mathrm{meV}$ and the electrical conductivity of $1 \times 10^{-1} \mathrm{~S} \mathrm{~cm}^{-1}$ at $298 \mathrm{~K} \cdot{ }^{65}$ Here, we measured the conductivity of pelletized NiAT, finding it to be close to an insulator with the activation energy of $113 \mathrm{meV}$ and the conductivity of $3 \times 10^{-6} \mathrm{~S} \mathrm{~cm}^{-1}$ at room temperature (Fig. S9†). This implies that NiAT functions as a redox switch of 
conductivity. The lower conductivity of NiAT was because there were no unpaired free electrons delocalized laterally in the sheet, in contrast to NiIT. The calculated band structures of NiIT (Fig. 5a) and NiAT (Fig. 5b) clearly show a typical kagome band, characterized by a set of Dirac bands and a flat band mostly derived from Ni- $\mathrm{d}_{x^{2}-y^{2}}$ orbitals. The band structure of NiIT indicates a metallic state with high electron density of states at the Fermi level, whereas that of NiAT shows an insulating state with the Fermi level lying inside a gap larger than $1.0 \mathrm{eV}$. The effect of adding one $\mathrm{H}$ atom per $\mathrm{N}$ atom to the NiAT sheet is to dope the system with electrons. There are six bands in NiIT between the Fermi level and the band gap above (shaded region in Fig. 5a), which are mainly ascribed to the $\mathrm{p}_{z}$ orbitals of $\mathrm{C}, \mathrm{N}$, and S. NiAT has 12 more electrons (one contributed by every $\mathrm{H}$ atom) than NiIT per unit cell, leading to all the six empty bands being filled, shifting the Fermi level into the band gap. In addition to doping electrons, after adding $\mathrm{H}$, the $\mathrm{N}$ valence orbitals are fully filled with a closed shell, so that electrons can hardly hop through the $\mathrm{N}$ sites. This effectively decreases the long-range hopping interaction between carbon rings, which in turn narrows the bandwidth and increases electron localization. Moreover, molecular orbitals by DFT calculation showed the frontier orbitals of NirT to be greatly delocalized over the entire ring, whereas those of NiAT are strongly localized (Fig. S11 $\dagger$ ). This also indicates much larger charge delocalization in NiIT than NiAT, consistent with the band structure calculation results. These theoretical results suggest that NiIT has a much higher electrical conductivity than NiAT, supporting the experimental observation.
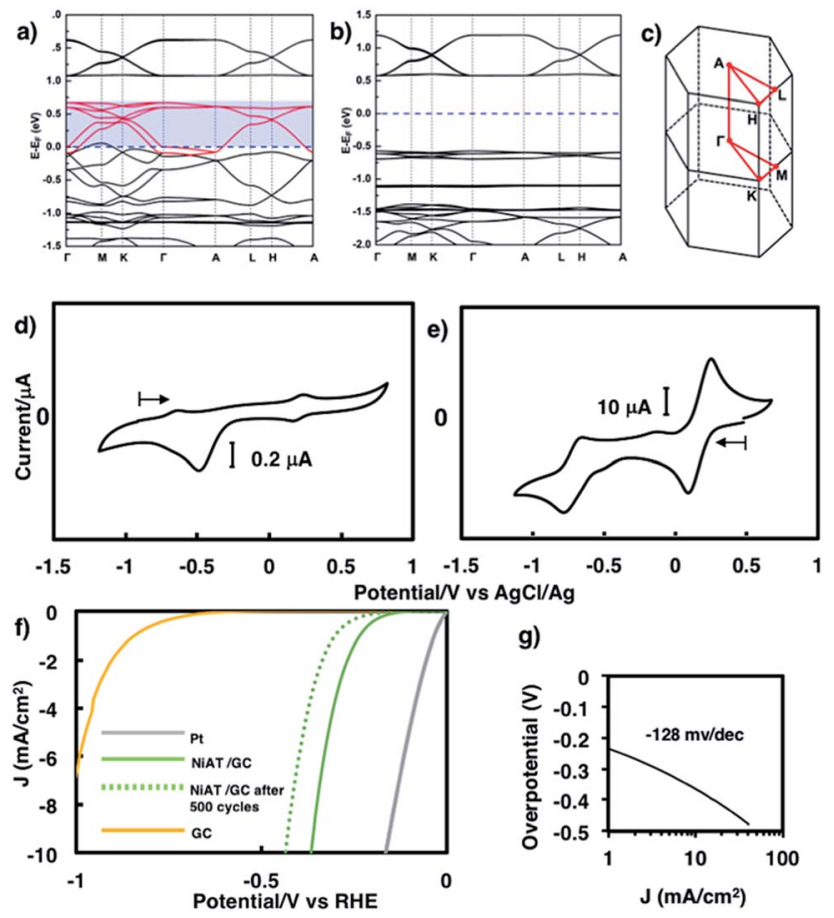

Fig. 5 First-principles band structures of (a) NiAT and (b) NiAT. (c) First Brillouin zone and high-symmetry $k$-points of NilT and NiAT. (d) Cyclic voltammogram of (d) NiAT and (e) NilT in $0.1 \mathrm{M} \mathrm{KCl}$ aq. at a scan rate of $50 \mathrm{mV} \mathrm{s}^{-1}$. (f) Linear sweep voltammograms for HER reaction with Pt, $\mathrm{NiAT} / \mathrm{GC}$, and $\mathrm{GC}$ in $0.05 \mathrm{M} \mathrm{H}_{2} \mathrm{SO}_{4}$ and (g) its Tafel slope.
Cyclic voltammograms of NiAT and NiIT in $0.1 \mathrm{M} \mathrm{KCl}$ are shown in Fig. 5d and e, respectively. NirT had two quasireversible pairs at 0.24 and $-0.66 \mathrm{~V}$ (Fig. 5e), suggesting that it underwent two successive, reversible one-electron reductions and two successive, one-electron oxidations. NiAT exhibited an irreversible peak at $-0.48 \mathrm{~V}$, probably caused by hydrogen migration from the amino group to the nickel centre to form a $\mathrm{Ni}-\mathrm{H}$ bond. ${ }^{66}$ The utilization of nanostructures as electrocatalysts for energy conversion technologies are intensively studied because their structures allowed for the sufficient exposure of well-defined active sites. ${ }^{55,58,63,64,67,68}$ The electrocatalytic activity of NiAT for the HER was examined because the NiAT-M complex exhibits higher catalytic ability for the HER than bis(diamino)nickel or bis(dithiolato)nickel. ${ }^{66}$ NiAT on glassy carbon (GC) protected with Nafion ${ }^{\circledR}$ was used as an electrocatalyst for the HER in acidic and neutral aqueous solutions. As the $\mathrm{pH}$ of the solution decreased, the catalytic efficiency increased (Fig. S12†). At pH =1.3, the onset potential for hydrogen evolution was $-0.15 \mathrm{~V} v s$. a reversible hydrogen electrode (RHE) with an operating potential of $-0.37 \mathrm{~V}$ at $10 \mathrm{~mA}$ $\mathrm{cm}^{-2}$ (Fig. 5f) and a Tafel slope of $128 \mathrm{mV} \mathrm{dec}^{-1}$ (Fig. 5g). The HER exchange current density $\left(j_{0}\right)$ for the NiAT catalysts obtained from the Tafel plots using the extrapolation method was $0.04 \mathrm{~mA} \mathrm{~cm}^{-2}$ (Fig. S13†). The Tafel slope in the plots is $128 \mathrm{mV}$ $\mathrm{dec}^{-1}$, which was further confirmed by electrochemical impedance spectroscopy (EIS, Fig. S14 $\dagger$ ). The charge-transfer Tafel slope derived from the linear fit of the plot of $\log R_{\mathrm{ct}}$

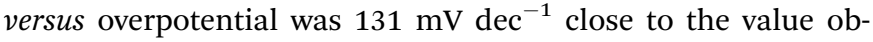
tained by the Tafel plots, indicating not only a charge transfer rate defining step but also a swift electron transfer characteristic of the NiAT catalyst in the HER. ${ }^{69}$ The turnover frequency (TOF), indicating the number of $\mathrm{H}_{2}$ molecules generated per second per active site, was calculated to be $0.1 \mathrm{~s}^{-1}$ at overpotential of $300 \mathrm{mV}$, using electrochemical surface area (ECSA, Fig. S15†) of the NiAT sheet by testing the electrochemical double layer capacitance $\left(C_{\mathrm{dl}}\right)$ (Fig. S16 $\dagger$ ). Compared with similar materials such as Co-benzenehexathiol and Cotriphenylenehexathiol complex nanosheets for HER catalysts, ${ }^{64}$ bis(aminothiolato)nickel motif outperformed bis(dithiolato) cobalt motif in many aspects. Moreover, NiAT exhibited relatively stable performance during 500 HER cycles (Fig. S17, $\uparrow$ potential cycling in the range of 0.27 to $-0.93 \mathrm{~V} v s$. RHE), XPS of NiAT showed that the component ratios and valence state remained unchanged after 500 HER cycles demonstrating the durability in acidic conditions (Fig. S18†). These results show that NiAT is an efficient, durable electrocatalytic cathode material for hydrogen generation from water.

\section{Conclusions}

In conclusion, we synthesized a crystalline CONASH containing NiAT moieties using the liquid-liquid and gas-liquid interfacial reactions. The morphology, composition, and crystal structure of NiAT were characterized by AFM, FE-SEM, XPS, ATR-IR, HRTEM, and GIXD. NiAT is an insulator and interconvertible to the conducting NiIT nanosheet by a proton-coupled redox reaction. These drastic changes in electrical conductivity were 
explained by the theoretical calculation of band structures. NiAT showed remarkable performance as an electrochemical HER catalyst. These results demonstrate a rare example of a CONASH acting as a redox switch for conductivity and as an electrocatalyst. Utilization for FET and other catalytic activities and energy storage abilities of this dual CONASH are currently being investigated.

\section{Methods}

\section{Materials}

All starting material and solvents were purchased from Tokyo Chemical Industry Co., Ltd. Dichloromethane was purified with a glass contour solvent dispensing system (Nikko Hansen \& Co., Ltd.). Water was purified using the Milli-Q purification system (Merck). 1,3,5-Triaminobenzene-2,4,6-trithiol (L), mononuclear complex bis(1-aminobenzene-2-thiolato)nickel(II) [NiAT-M], mononuclear complex bis(1-iminobenzene-2-thiolato)nickel(II) $\left[\right.$ NiIT-M] and were prepared according to the literature. ${ }^{66,70}$

\section{Substrate preparation}

HOPG was purchased from Alliance Biosystems, Inc. (Grade SPI$110 \times 10 \times 2 \mathrm{~mm}$ ) and cleaved with adhesive tape prior to use. Silicon wafers ( $\mathrm{p}$-doped with a carrier concentration of $3 \times 10^{18}$ $\mathrm{cm}^{-3}$ and resistivity of 1-10 $\mathrm{ohm} \mathrm{cm}$ ) with thermally-grown 100 nm-thick $\mathrm{SiO}_{2}$ were purchased from Yamanaka Semiconductor and cut into squares $(10 \times 10 \mathrm{~mm})$. 1,1,1,3,3,3-Hexamethyldisilanaze (HMDS)-modified $\mathrm{SiO}_{2} / \mathrm{Si}$ substrates were prepared by depositing $\mathrm{HMDS}$ on $\mathrm{SiO}_{2} / \mathrm{Si}$ substrates, keeping them under vacuum for $10 \mathrm{~min}$, and then placing them in a flow of Ar. The HMDS-modified $\mathrm{SiO}_{2} / \mathrm{Si}$ substrates were kept in anhydrous ethanol.

\section{Preparation of multi-layer NiAT}

Under an Ar atmosphere, a degassed aqueous solution $(10 \mathrm{~mL})$ containing $2.4 \times 10^{-5} \mathrm{~mol} \mathrm{~L}^{-1}$ of $\mathbf{L}$, and organic layer of a dichloromethane solution of $0.5 \mathrm{mM} \mathrm{Ni}(\text { acac })_{2}(10 \mathrm{~mL})$. The organic layer was overlaid slowly with the aqueous $\mathbf{L}$ solution. After standing in an Ar atmosphere for 10 days, a yellow nanosheet was obtained at the aqueous/organic interface. After the removal of the aqueous and organic phases, multi-layer NiAT was washed thoroughly with water, ethanol, and dichloromethane, and dried in vacuo at $120^{\circ} \mathrm{C}$. NiAT could be oxidized under long time explosion into air, thus all characterizations and measurements took suitable oxygen segregation treatments. Elemental analysis for $\mathrm{NiC}_{4} \mathrm{H}_{4} \mathrm{~N}_{2} \mathrm{~S}_{2}$ : calcd: C: $23.66 \% ; \mathrm{H}$ : $2.02 \%$; N: $13.81 \%$. Found: C: $23.36 \%$; H: $2.32 \%$; N: $14.12 \%$.

\section{Preparation of single-layer NiAT}

A dilute dichloromethane $(10 \mu \mathrm{L})$-ethyl acetate $(1 \mu \mathrm{L})$ solution of $\mathrm{Ni}(\mathrm{acac})_{2}(0.5 \mathrm{mM})$ was gently dropped onto an aqueous solution of $\mathbf{L}(35 \mu \mathrm{M})$ at ambient temperature using a micro syringe. After spontaneous evaporation of the organic solvent, the reaction system was left undisturbed, so that single-layer NiAT was produced at the air-liquid interface. The nanosheet was then transferred onto substrates using the Langmuir-Schaefer method.

\section{Characterization}

Optical microscope images were taken using VHX-100 (Keyence Corporation). FE-SEM images were collected using a scanning electron microscope (JSM-7400 FNT, JEOL). The samples were prepared by depositing an ethanol suspension of NiAT on HMDS-modified $\mathrm{SiO}_{2} / \mathrm{Si}$ substrates. TEM images were recorded with a transmission electron microscope (HF-2000, Hitachi) equipped with a AMT-CCD camera (HISCO, AMT552) at $75 \mathrm{kV}$. The TEM samples were prepared by depositing an ethanol suspension of NiAT on a carbon film supported by a copper grid. GIXD data were obtained using synchrotron radiation $(\lambda=$ $1.00 \AA)$ at BL45XU in SPring-8, Japan. Diffractions from a sample were detected using a Pilatus3X $2 \mathrm{M}$ detector. Recorded diffraction images were integrated along the Debye-Scherrer ring using a FIT2D software, affording a one-dimensional intensity profile. To prepare the samples, NiAT was deposited on HMDS-modified $\mathrm{SiO}_{2} / \mathrm{Si}$ substrates. ATR-IR spectra were recorded using an IR spectrometer (FT/IR-6100, JASCO) at room temperature under vacuum. The samples were prepared by depositing NiAT on HMDS-modified $\mathrm{SiO}_{2} / \mathrm{Si}$ substrates. XPS data were obtained using an XPS microprobe (PHI 5000 VersaProbe, ULVAC-PHI, Inc.). Al K $\alpha(15 \mathrm{kV}, 25 \mathrm{~W})$ was used as the $\mathrm{X}$-ray source, and the beam was focused on a $100 \mu \mathrm{m}^{2}$ area. The spectra were analyzed with MultiPak (MultiPak Version 9.2.0.5 ULVAC-PHI, Inc.), and standardized using the C (1s) peak at $284.8 \mathrm{eV}$. Atomic force microscopy measurements were carried out using a scanning probe microscope (5500, Agilent Technologies) under ambient conditions high-amplitude mode (tapping mode) with a silicon cantilever probe (PPP-NCL, Nano World).

\section{SAED and GIXD simulation}

To reproduce the obtained SAED and 2D WAXS pattern (Fig. 3d), two types of $3 \mathrm{D}$ lattices comprising piles of single-layer NiAT were considered. The two lattices include eclipsed (AA) and staggered $\left(\mathrm{AA}^{-1}\right)$ stack models, because 6-fold symmetry is required to reproduce the hexagonal diffraction pattern in SAED. First, the atomic arrangement of single-layer NiAT was optimized using density-functional theory implemented in performed on Gaussian 09: ${ }^{71}$ all the optimized structures were obtained at the B3LYP level of theory. As the basis sets, 6-31G* is used. The optimized cell was then subjected so as to reproduce the observed diffraction data. The eclipsed and staggered stack models were then constructed by using space group of $P 3$ and $P \overline{3}$, respectively. Slipped-parallel (AB) orientations pattern simulations were performed using eclipsed (AA) stack models fixing an interlayer separation of $0.42 \mathrm{~nm}$ with different stacking angles $\beta$. The SAED and GIXD patterns were simulated by implementing CrystalMaker 2.6.3, SingleCrystal 2.3, and CrystalDiffract 6.5.5 (CrystalMaker Software Ltd).

\section{Electrical properties}

Electrical conductivity data were collected using the van der Pauw method. ${ }^{72}$ NiAT $(2.1 \mathrm{mg}$ ) prepared using conditions of described in Preparation of multi-layer NiAT were grinded then 
pressed with a pressure of $500 \mathrm{~kg} \mathrm{~cm}^{-2}$ to form self-standing films. The pelletized films were placed on fluoropolymer boards and attached to gold wires using carbon paste (Fujikura Kasei Co., Ltd.). The $\mathrm{O}_{2}$ prevention procedures are applied to every subjectable step.

\section{Band structure calculation}

The first-principles band structure calculations based on density-functional-theory method were performed in the framework of the projector augmented wave approach and the generalized gradient approximation of Perdew-Burke-Ernzerhof for the exchange-correlation potentials, as implemented in Vienna $a b$ initio simulation package. ${ }^{73,74}$ Self-consistent calculations were carried out with an energy cut off of $400 \mathrm{eV}$ on a $5 \times$ $5 \times 7 \Gamma$-centered $K$-point mesh. All atoms were fully relaxed in the structural optimizations until the atomic forces were smaller than $0.01 \mathrm{eV}^{-1}$. Electron spin polarization was also considered. The experimental lattice constants of NiAT and NiIT with AB-stacking pattern were adopted for the band structure calculations.

\section{Electrochemical methods}

Electrochemistry experiments were carried out using an electrochemical analyzer (650DT, ALS). A platinum wire served as the auxiliary electrode, and the reference electrode was an $\mathrm{Ag} /$ $\mathrm{AgCl}$ electrode (silver wire immersed in $0.1 \mathrm{M} \mathrm{Bu}_{4} \mathrm{NClO}_{4} /$ $\left.0.01 \mathrm{M} \mathrm{AgClO} / \mathrm{CH}_{3} \mathrm{CN}\right)$, calibrated with a ferrocenium/ ferrocene redox potential. To make the working electrode, NiAT $(1.7 \mathrm{mg}$ ) was placed on $10 \mathrm{~mm} \phi$ aluminum foil to cover entire surface, another $10 \mathrm{~mm} \phi$ carbon paper was put on top to make a "sandwich", then pressed with a pressure of $500 \mathrm{~kg}$ $\mathrm{cm}^{-2}$. For the HER, a $3 \mathrm{~mm} \phi$ GC working electrode was used. Before use, the GC electrodes were polished using aqueous alumina suspensions on felt polishing pads. The potentials for the HER in this study refer to those of the reversible hydrogen electrode (RHE) obtained by adding E(SCE) + $0.059 \mathrm{pH}$. The aqueous solutions used in the electrochemical experiments were prepared as follows. For cyclic voltammograms of NIAT, $\mathrm{KCl}(0.1493 \mathrm{~g})$ was dissolved in water $(20 \mathrm{~mL})$ to make $0.1 \mathrm{M} \mathrm{KCl}$ aqueous solution. For the $\mathrm{pH} 1.3$ solution, $\mathrm{H}_{2} \mathrm{SO}_{4}(0.0534 \mathrm{~mL}, c$ $=98 \%)$ was dissolved in water $(20 \mathrm{~mL})$. The solutions were deoxygenated with pure Ar before measurement. The catalyst ink was prepared by suspending NiAT $(0.2 \mathrm{mg})$ in ethanol (4 $\mathrm{mL}$ ). The catalyst ink was pipetted onto the GC surface to achieve the desired catalyst loading. Then, $7 \mu \mathrm{L}$ of $5 \%$ Nafion ${ }^{\circledR}$ solution in ethanol was drop-cast on top to protect the NiAT film. The HER was measured by cyclic voltammetry with a sweep rate of $50 \mathrm{mV} \mathrm{s}^{-1}$. For comparison, the same GC electrode was re-polished after drop casting the catalyst, redrop the same amount of Nafion without catalyst and measured in the same aqueous solution to give a naked GC HER result. Moreover, a $2 \mathrm{~mm} \phi$ Pt electrode was also measured for comparison.

\section{Conflicts of interest}

There are no conflicts of interest to declare.

\section{Acknowledgements}

This work is supported by JST-CREST "Development of Atomic or Molecular Two-Dimensional Functional Films and Creation of Fundamental Technologies for Their Applications" (JPMJCR15F2) and JSPS KAKENHI Grant Numbers JP15H00862, JP15J06673, JP15K13654, JP16H00900, JP16H00957, JP26220801, JP26708005, JP17H05354, JP17H03028. Work at Utah is supported by US Department of Energy (Grant No. DE FG02-04ER46148). The synchrotron radiation experiments were performed at BL45XU in SPring-8 with the approval of RIKEN (Proposal No. 20160041). XPS measurements were conducted at Advanced Characterization Nanotechnology Platform of the University of Tokyo, supported by "Nanotechnology Platform" of the Ministry of Education, Culture, Sports, Science and Technology (MEXT), Japan. We acknowledge NERSC and CHPC at the University of Utah for providing the computing resources.

\section{References}

1 K. S. Novoselov, A. K. Geim, S. V. Morozov, D. Jiang, Y. Zhang, S. V. Dubonos, I. V. Grigorieva and A. A. Firsov, Science, 2004, 306, 666.

2 A. C. Ferrari, F. Bonaccorso, V. Fal'ko, K. S. Novoselov, S. Roche, P. Boggild, S. Borini, F. H. L. Koppens, V. Palermo, N. Pugno, J. A. Garrido, R. Sordan, A. Bianco, L. Ballerini, M. Prato, E. Lidorikis, J. Kivioja, C. Marinelli, T. Ryhanen, A. Morpurgo, J. N. Coleman, V. Nicolosi, L. Colombo, A. Fert, M. Garcia-Hernandez, A. Bachtold, G. F. Schneider, F. Guinea, C. Dekker, M. Barbone, Z. Sun, C. Galiotis, A. N. Grigorenko, G. Konstantatos, A. Kis, M. Katsnelson, L. Vandersypen, A. Loiseau, V. Morandi, D. Neumaier, E. Treossi, V. Pellegrini, M. Polini, A. Tredicucci, G. M. Williams, B. Hee Hong, J.-H. Ahn, J. Min Kim, H. Zirath, B. J. van Wees, H. van der Zant, L. Occhipinti, A. Di Matteo, I. A. Kinloch, T. Seyller, E. Quesnel, X. Feng, K. Teo, N. Rupesinghe, P. Hakonen, S. R. T. Neil, Q. Tannock, T. Lofwander and J. Kinaret, Nanoscale, 2015, 7, 4598.

3 L. Wang and T. Sasaki, Chem. Rev., 2014, 114, 9455.

4 R. Ma and T. Sasaki, Acc. Chem. Res., 2015, 48, 136.

5 S.-L. Li, K. Tsukagoshi, E. Orgiu and P. Samori, Chem. Soc. Rev., 2016, 45, 118.

6 R. Lv, J. A. Robinson, R. E. Schaak, D. Sun, Y. Sun, T. E. Mallouk and M. Terrones, Acc. Chem. Res., 2015, 48, 56. 7 M. Bosi, RSC Adv., 2015, 5, 75500.

8 G. Abellań, C. Marti-Gastaldo, A. Ribera and E. Coronado, Acc. Chem. Res., 2015, 48, 160.

9 Q. Wang and D. O'Hare, Chem. Rev., 2012, 112, 4124.

10 R. Ma, Z. Liu, L. Li, N. Iyi and T. Sasaki, J. Mater. Chem., 2006, 16, 3809.

11 P. J. Waller, F. Gandara and O. M. Yaghi, Acc. Chem. Res., 2015, 48, 3053.

12 K. Sakaushi and M. Antoniettie, Acc. Chem. Res., 2015, 48, 1591.

13 M. Dogru and T. Bein, Chem. Commun., 2014, 50, 5531. 
14 X.-H. Liu, C.-Z. Guan, W. Dong and L.-J. Wan, Adv. Mater., 2014, 26, 6912.

15 Q. H. Wang, K. Kalantar-Zadeh, A. Kis, J. N. Coleman and M. S. Strano, Nat. Nanotechnol., 2012, 7, 699.

16 R. Cheng, S. Jiang, Y. Chen, Y. Liu, N. Weiss, H.-C. Cheng, H. Wu, Y. Huang and X. Duan, Nat. Commun., 2014, 5, 5143.

17 M. C. Lemme, T. J. Echtermeyer, M. Baus and H. Kurz, IEEE Electron Device Lett., 2007, 28, 282.

18 F. Schedin, A. K. Geim, S. V. Morzov, E. W. Hill, P. Blake, M. I. Katsnelson and K. S. Novoselov, Nat. Mater., 2007, 6, 652.

19 J. Yan, M.-H. Kim, J. A. Elie, A. B. Sushkov, G. S. Jenkins, H. M. Milchberg, M. S. Fuhrer and H. D. Drew, Nat. Nanotechnol., 2012, 7, 472.

20 Y. D. Kim, H. Kim, Y. Cho, J. H. Ryoo, C.-H. Park, P. Kim, Y. S. Kim, S. Lee, Y. Li, S.-N. Park, Y. S. Yoo, D. Yoon, V. E. Dorgan, E. Pop, T. F. Heinz, J. Hone, S.-H. Chun, H. Cheong, S. W. Lee, M.-H. Bae and Y. D. Park, Nat. Nanotechnol., 2015, 10, 676.

21 Y. J. Zhang, T. Oka, R. Suzuki, J. T. Ye and Y. Iwasa, Science, 2014, 344, 725.

22 S. Mouri, Y. Miyauchi and K. Matsuda, Nano Lett., 2013, 13, 5944.

23 Q. Liang, X. Yao, W. Wang, Y. Liu and C. P. Wong, ACS Nano, 2011, 5, 2392.

24 K. M. F. Shahil and A. A. Balandin, Nano Lett., 2012, 12, 861.

25 G. C. Rodrigues, P. Zelenovskiy, K. Romanyuk, S. Luchkin, Y. Kopelevich and A. Kholkin, Nat. Commun., 2014, 6, 7572. 26 M. T. Ong and E. J. Reed, ACS Nano, 2012, 6, 1387.

27 W. Wu, L. Wang, Y. Li, F. Zhang, L. Lin, S. Niu, D. Chenet, X. Zhang, Y. Hao, T. F. Heinz, J. Hone and Z. L. Wang, Nature, 2014, 514, 470.

28 A. Dmitriev, H. Spillmann, N. Lin, J. V. Barth and K. Kern, Angew. Chem., Int. Ed., 2003, 42, 2670.

29 W. Xu, J.-G. Wang, M. Yu, E. Laegsgaard, I. Stensgaard, T. R. Linderoth, B. Hammer, C. Wang and F. Besenbacher, J. Am. Chem. Soc., 2010, 132, 15927.

30 Y. Li, J. Xiao, T. E. Shubina, M. Chen, Z. Shi, M. Schmid, H.-P. Steinrück, M. Gottfried and N. Lin, J. Am. Chem. Soc., 2012, 134, 6401.

31 C. S. Kley, J. Čechal, T. Kumagai, F. Schramm, M. Ruben, S. Stepanow and K. Kern, J. Am. Chem. Soc., 2012, 134, 6072. 32 Z. Shi and N. Lin, J. Am. Chem. Soc., 2009, 131, 5376.

33 J. Liu, T. Lin, Z. Shi, F. Xia, L. Dong, P. N. Liu and N. Lin, J. Am. Chem. Soc., 2011, 133, 18760.

34 H. Walch, J. Dienstmaier, G. Eder, R. Gutzler, S. Schlögl, T. Sirtl, K. Das, M. Schmittel and M. Lackinger, J. Am. Chem. Soc., 2011, 133, 7909.

35 T.-C. Tseng, N. Abdurakhmanova, S. Stepanow and K. Kern, J. Phys. Chem. C, 2011, 115, 10211.

36 Z. Shi and N. Lin, J. Am. Chem. Soc., 2010, 132, 10756.

37 M. Matena, M. Stöhr, T. Riehm, J. Björk, S. Martens, M. S. Dyer, M. Persson, J. Lobo-Checa, K. Müller, M. Enache, H. Wadepohl, J. Zegenhagen, T. A. Jung and L. H. Gade, Chem.-Eur. J., 2010, 16, 2079.

38 J. A. A. W. Elemans, S. Lei and S. D. Feyter, Angew. Chem., Int. Ed., 2009, 48, 7298.
39 S. Motoyama, R. Makiura, O. Sakata and H. Kitagawa, J. Am. Chem. Soc., 2011, 133, 5640.

40 R. Makiura, S. Motoyama, Y. Umemura, H. Yamanaka, O. Sakata and H. Kitagawa, Nat. Mater., 2010, 9, 565.

41 S. Stepanow, N. Lin, D. Payer, U. Schlickum, F. Klappenberger, G. Zoppellaro, M. Ruben, H. Brune, J. V. Barth and K. Kern, Angew. Chem., Int. Ed., 2007, 46, 710. 42 T. Kambe, T. Kusamoto, R. Sakamoto and H. Nishihara, Macromol. Symp., 2015, 351, 78.

43 K. Takada, R. Sakamoto, S.-T. Yi, S. Katagiri, T. Kambe and

H. Nishihara, J. Am. Chem. Soc., 2015, 137, 4681.

44 R. Sakamoto, K. Hoshiko, Q. Liu, T. Yagi, T. Nagayama, S. Kusaka, M. Tsuchiya, Y. Kitagawa, W.-Y. Wong and H. Nishihara, Nat. Commun., 2015, 6, 6713.

45 R. Sakamoto, T. Iwashima, M. Tsuchiya, R. Toyoda, R. Matsuoka, J. F. Kögel, S. Kusaka, K. Hoshiko, T. Yagi, T. Nagayama and H. Nishihara, J. Mater. Chem. A, 2015, 3, 15357.

46 R. Sakamoto, K. Takada, X. Sun, T. Pal, T. Tsukamoto, E. J. H. Phua, A. Rapakousiou, K. Hoshiko and H. Nishihara, Coord. Chem. Rev., 2016, 320-321, 118.

47 H. Maeda, R. Sakamoto and H. Nishihara, Langmuir, 2016, 32, 2527.

48 T. Kambe, R. Sakamoto, K. Hoshiko, K. Takada, M. Miyachi, J. H. Ryu, S. Sasaki, J. Kim, K. Nakazato, M. Takata and H. Nishihara, J. Am. Chem. Soc., 2013, 135, 2462.

49 M. Hmadeh, Z. Lu, Z. Liu, F. Gándara, H. Furukawa, S. Wan, V. Augustyn, R. Chang, L. Liao, F. Zhou, E. Perre, V. Ozolins, K. Suenaga, X. Duan, B. Dunn, Y. Yamamto, O. Terasaki and O. M. Yaghi, Chem. Mater., 2012, 24, 3511.

50 M. G. Campbell, S. F. Liu, T. M. Swager and M. Dincă, J. Am. Chem. Soc., 2015, 137, 13780.

51 K. Hoshiko, T. Kambe, R. Sakamoto, K. Takada and H. Nishihara, Chem. Lett., 2014, 43, 252.

52 T. Pal, T. Kambe, T. Kusamoto, M. L. Foo, R. Matsuoka, R. Sakamoto and H. Nishihara, ChemPlusChem, 2015, 80, 1255.

53 X. Huang, P. Sheng, Z. Tu, F. Zhang, J. Wang, H. Geng, Y. Zou, C.-a. Di, Y. Yi, Y. Sun, W. Xu and D. Zhu, Nat. Commun., 2015, 6, 7408.

54 D. Sheberla, L. Sun, M. Blood-Forsythe, S. Er, C. R. Wade, C. K Brozek, A. Aspuru-Guzik and M. Dincă, J. Am. Chem. Soc., 2014, 136, 8859.

55 E. M. Miner, T. Fukushima, D. Sheberla, L. Sun, Y. Surendranath and M. Dincă, Nat. Commun., 2015, 7, 10942.

56 D. Sheberla, J. C. Bachman, J. S. Elias, C.-J. Sun, Y. ShaoHornand and M. Dincă, Nat. Mater., 2016, 16, 220.

57 M. G. Campbell, D. Sheberla, S. F. Liu, T. M. Swager and M. Dincă, Angew. Chem., Int. Ed., 2015, 54, 4349.

58 R. Dong, M. Pfeffermann, H. Liang, Z. Zheng, X. Zhu, J. Zhang and X. Feng, Angew. Chem., Int. Ed., 2015, 54, 12058. 59 J. Cui and Z. Xu, Chem. Commun., 2014, 50, 3986.

60 N. Lahiri, N. Lotfizadeh, R. Tsuchikawa, V. Deshpande and J. Louie, J. Am. Chem. Soc., 2017, 139, 19.

61 T. Kambe, R. Sakamoto, T. Kusamoto, T. Pal, N. Fukui, K. Hoshiko, T. Shimojima, Z. Wang, T. Hirahara, 
K. Ishizaka, S. Hasegawa, F. Liu and H. Nishihara, J. Am. Chem. Soc., 2014, 136, 14357.

62 Z. F. Wang, N. Su and F. Liu, Nano Lett., 2013, 13, 2842.

63 R. Dong, Z. Zheng, D. C. Tranca, J. Zhang, N. Chandrasekhar, S. Liu, X. Zhuang, G. Seifert and X. Feng, Chem.-Eur. J., 2016, 23, 2255.

64 A. J. Clough, J. W. Yoo, M. H. Mecklenburg and S. C. Marinescu, J. Am. Chem. Soc., 2015, 137, 118.

65 X. Sun, K.-H. Wu, R. Sakamoto, T. Kusamoto, H. Maeda and H. Nishihara, Chem. Lett., 2017, 46, 1072.

66 A. Das, Z. Han, W. W. Brennessel, P. L. Holland and R. Eisenberg, ACS Catal., 2015, 5, 1397.

67 J. Hu, C. Zhang, X. Meng, H. Lin, C. Hu, X. Long and S. Yang, J. Mater. Chem. A, 2017, 5, 5995.
68 Y. An, B. Huang, Z. Wang, X. Long, Y. Qiu, J. Hu, D. Zhou, H. Lin and S. Yang, Dalton Trans., 2017, 46, 10700.

69 J. Hu, B. Huang, C. Zhang, Z. Wang, Y. An, D. Zhou, H. Lin, M. K. H. Leung and S. Yang, Energy Environ. Sci., 2017, 10, 593.

70 G. Grandolini and A. Martani, Gazz. Chim. Ital., 1962, 92, 1150.

71 M. J. Frisch, et al., Gaussian 09, Revision B.01, Gaussian Inc., Wallingford CT, 2010.

72 L. J. van der Pauw, Technol. Rev., 1958, 20, 220.

73 P. E. Blöchl, Phys. Rev. B, 1994, 50, 17953.

74 J. P. Perdew, K. Burke and M. Ernzerhof, Phys. Rev. Lett., 1996, 77, 3865. 